\title{
Corneal sensation in scleritis and episcleritis
}

\author{
A. J. LYNE \\ From the Scleritis Clinic, Department of Clinical Ophthalmology, Institute of Ophthalmology, \\ Moorfields Eye Hospital, City Road, London
}

SUMMARY A series of 59 patients were examined for loss of corneal sensitivity. Thirty-five of them suffered from scleritis and 24 suffered from episcleritis. There was a difference between the 2 conditions in that significantly more patients with scleritis were affected than patients with episcleritis. It was also found that among the scleritis patients there was a close relationship between the number of quadrants affected and decrease in sensitivity. Five patients had necrotising scleritis, and they all showed marked loss of sensitivity. Sensitivity returned to normal when the scleritis resolved except when a large area of scleral ectasia resulted.

The test is of importance because it is one of the easiest methods of detecting the onset of necrotising scleritis in its earliest stages.

Episcleritis is a benign disease, causing no rermanent damage to the eye and requiring only local therapy to hasten its resolution. Scleritis is a disease which, although varying greatly in severity, can be blinding on occasion. It requires systemic therapy and careful supervision in order to avoid the diastrous complications which may supervene.

It can be difficult at times to distinguish between the two conditions, especially when they are seen in the early stages, and it is at this stage that it is desirable to diagnose scleritis so that early treatment can be instituted. The presence of pain as opposed to discomfort, the vascular pattern, the appearance of scleral transparency, and the response to treatment are all of help when assessing a patient (Watson and Hazleman, 1976). Any additional clinical test that would be of value in distinguishing between episcleritis and scleritis would therefore be welcome.

It was thought that corneal sensation might be affected differently in the two conditions. Scleritis is usually a granulomatous condition destroying the sclera where the collagen fibres are more closely arranged, while episcleritis is an inflammatory condition in vascular tissues where collagen fibres of the episclera are more loosely arranged. The majority of nerve fibres subserving corneal sensation pass forwards between the sclera and choroid until they branch and pass through the sclera to the region of the limbus to form the perilimbal plexus. It would seem likely that scleritis would be more likely to cause swelling of the scleral fibres and

Address for reprints: A. J. Lyne, FRCS, Peterborough District Hospital, Peterborough, Cambridgeshire consequent compression and possible destruction of the nerve fibres than would episcleritis. It is also known that corneal sensation may be profoundly affected in cases of sclerokeratitis due to herpes zoster, although other factors are operative here (Marsh, 1973).

In order to investigate the possible decrease in sensation in the two disorders a total of 59 patients attending the Scleritis Clinic at Moorfields Eye Hospital, City Road, London, suffering from scleritis and episcleritis were examined.

\section{Methods}

The instrument used to examine the corneal sensation was the corneal aesthesiometer of Cochet and Bonnet (Fig. 1). This instrument is based on the principle first described by von Frey and modified later by Boberg-Ans (Boberg-Ans, 1955). The instrument has many disadvantages but it does have the virtue of extreme portability and ease of manipulation. It consists of a filament of nylon contained in a holder, one end of the filament projecting out. The length of the projecting portion can be controlled by a small milled wheel on the handle. The instrument works on the principle that the longer the length of filament projecting, the less the pressure required to bend it.

The patient was placed in a reclining chair and was instructed to look at a point on the ceiling. The instrument was set on its longest length, which is $6 \mathrm{~cm}$, and was slowly brought down to the corneal surface, ensuring that it was applied at right-angles to the cornea. The filament was pressed 


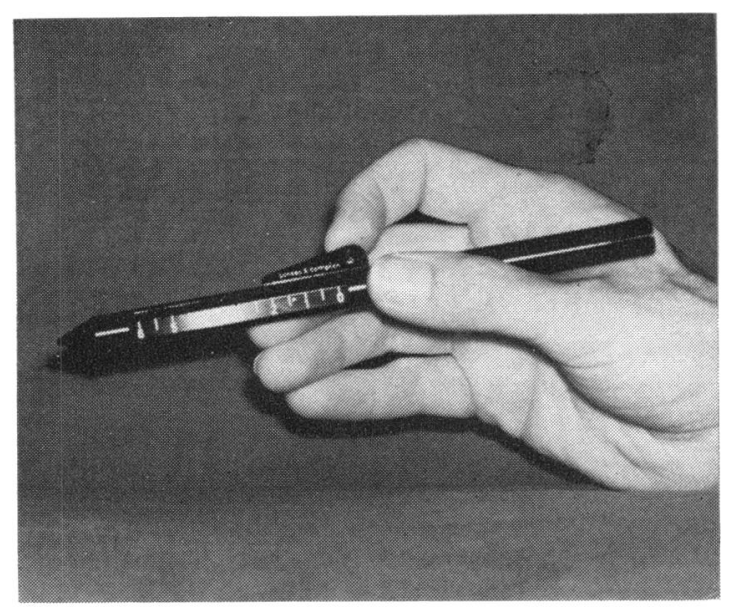

Fig. 1 The corneal aesthesiometer of Cochet and Bonnet

on the cornea until it could be seen to bend and the patient was asked to say if he felt anything. If he did, the filament length was recorded as $6 \mathrm{~cm}$ and the test repeated. Three tests on each of the points of the cornea to be described were performed on each occasion, and the result was recorded as positive if 2 out of the 3 were felt. If the patient felt nothing the filament was shortened by $1 \mathrm{~cm}$ and the test repeated. The filament was shortened by a centimetre progressively until the stimulus was felt, and the length of filament was then recorded in centimetres.

The corneal sensation at the centre of the cornea and in the 4 quadrants was tested as shown (Fig. 2).

The results of the test on each eye were recorded on a chart as shown (Fig. 3). It will be seen that, the lower the number of figures obtained in each region, the less acute the corneal sensitivity. Conversely, the higher the recorded number the greater the sensitivity.

The quadrants chosen were the temporal, nasal, superior, and inferior rather than superotemporal, inferotemporal, superonasal, and inferonasal, because it was felt that the nasal and temporal situation

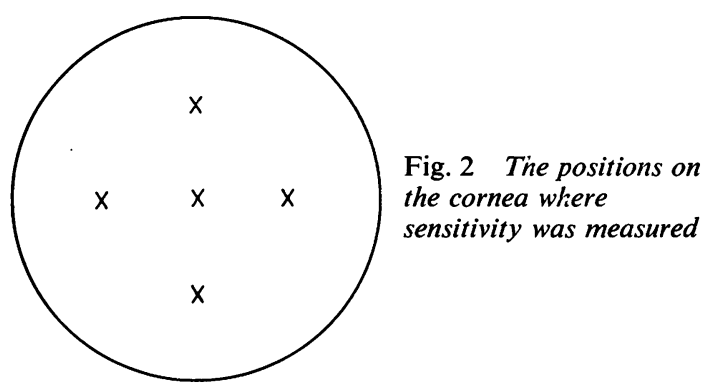

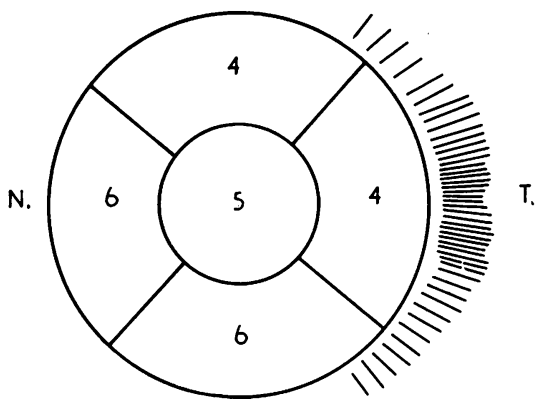

Fig. 3 The charting of corneal sensitivities, with an area of inflammation recorded on the temporal side

of the long ciliary nerves might ke relevant. In fact it is doubtful if there would have been any difference in the findings.

On the same chart the distribution of the scleritis was recorded by radial lines. The closer the lines were together the greater the intensity of the inflammation. A special note was made of areas of necrotising scleritis if present because it was felt that the latter was of particular importance. Cornea involved by sclerosing keratitis was indicated and staphylomata were also recorded.

In this investigation the corneal sensation of one eye was compared with the other so that factors such as humidity, which affects the elasticity of the nylon filament, diurnal variation of sensitivity (Millodot, 1972), menstruation (Millodot and Lamont, 1974), and age (Boberg-Ans, 1956) did not have to be considered. Testing in this way had the disadvantage that bilateral cases of scleritis and episcleritis could not be included in the trial.

In order to get consistent results with the instrument testing must be done with care, and particular attention must be paid to the following points: (a) The instrument must be applied at right-angles to the cornea. If this is not done the end of the filament skids across the corneal surface and variable answers are given by the patient. (b) Likewise, the instument must be held steady. If it is not the tip of the filament describes an ellipse on the cornea, which increases the stimulus. It is easy to keep the filament steady when it is short but by no means so when it is at its maximum length. (c) The lashes must not be touched by the tip or a positive response is immediately given. $(d)$ The filament must approach the point to be tested from the side, otherwise it is seen by the patient and he gives a positive response almost before it touches the eye. (e) The filament must be applied slowly and steadily. (f) It is important that the stimulus is applied exactly half way between the centre of the cornea and the limbus in the 4 quadrants. Corneal sensation 
is normally most acute in the centre of the cornea and falls off rapidly as the limbus is approached. If the stimulus were to be applied at differing distances from the centre of the cornea widely different results would be obtained.

\section{Results}

A total of 59 patients were examined, 35 suffering from scleritis and 24 from episcleritis. The preponderance of patients with scleritis does not reflect the incidence of these conditions in the population but rather the fact that episcleritis tends to be treated successfully by the general practitioner and casualty department whereas the scleritis patients end up in a special clinic.

The results were charted by adding the numbers obtained in each quadrant of each eye together and then comparing the total for 1 eye with the other. It was thought that only a significant difference between the 2 eyes should be recorded as positive if the investigation was to be of value. The results obtained are subjective on the part of the patient, and patients vary greatly in their ability to give consistent responses. A difference of 3 or more was taken arbitrarily as being significant.

Out of the 35 patients suffering from scleritis 21 showed a decrease in corneal sensation. Out of the 24 patients suffering from episcleritis only 5 showed decreased sensitivity (Fig. 4). There is a significant difference in corneal sensation between the two conditions $(\mathrm{P}<0.05)$.

When the patients with scleritis were analysed in more detail other interesting findings were revealed. It was found that the amount of perilimbal circumference involved was a significant factor. It seemed that a certain proportion of the perilimbal area needed to be affected before any effect upon sensation occurred. Many of these patients were examined during and after each recurrence in order to record recovery or otherwise of their corneal sensitivity.

The area of sclera involved was charted by means of the simple diagram already described and the number of quadrants involved recorded. It was

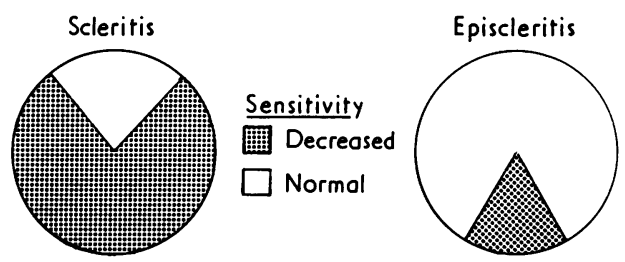

21 offected out of 35 patients

5 affected out of 24 patients

Fig. 4 Number of patients with decreased corneal sensation in scleritis and episcleritis
More than one quadrant involved Less than one quadrant involved

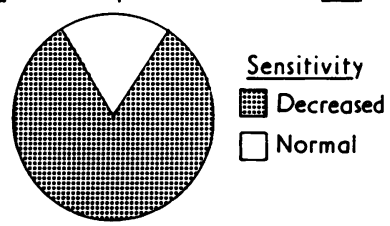

12 offected out of
15 potient episodes

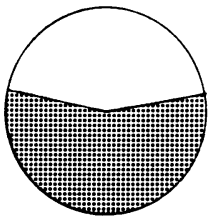

27 affected out of
Fig. 5 Effect of the number of quadrants involved by scleritis on reduction in corneal sensitivity

found that those patients in which more than 1 quadrant was involved showed a greater incidence of decreased sensation than those in whom only 1 quadrant was involved. There were 27 episodes involving 1 quadrant and 13 of these showed decreased sensitivity, as compared with 15 episodes involving more than 1 quadrant in which 12 showed decreased sensitivity (Fig. 5) (statistically significant, $P<0.05$ ).

In this aspect of the study patients with necrotising scleritis were omitted because profound loss of sensation in this condition occurs irrespective of the number of quadrants involved.

In all patients the corneal sensitivity returned to normal when the inflammation subsided except when a staphyloma resulted, and even then it did so if the staphyloma was small. Sclerosing keratitis appeared to have no effect on sensitivity.

\section{Discussion}

It is perhaps not surprising that corneal sensitivity is not often affected in episcleritis (5 patients out of 24), since this condition is a superficial inflammation and not likely to affect nerves that are lying at a deeper level. It does seem odd, however, that sensitivity is not affected more often in scleritis (21 patients out of 35). The explanation may lie in the anatomical arrangement of the corneal nerve supply.

Accounts of this vary in detail but the consensus is that it is derived from the long ciliary nerves ( 2 in number) and the short ciliary nerves (10 to 12 in number). All of them enter the eye by piercing the scleral coat posteriorly and passing forwards in the suprachoroidal space to the ciliary body to form the ciliary plexus in the ciliary muscle. Branches from this go to supply the ciliary muscle itself, the iris, the sclera, and the cornea. The corneal nerves divide and inosculate to form an annular plexus at the limbus. From the perilimbal plexus 60 to 80 nerves run towards the centre of the cornea, lying in its posterior third and dividing dichotomously as they go. A further network of nerves lies at the level 


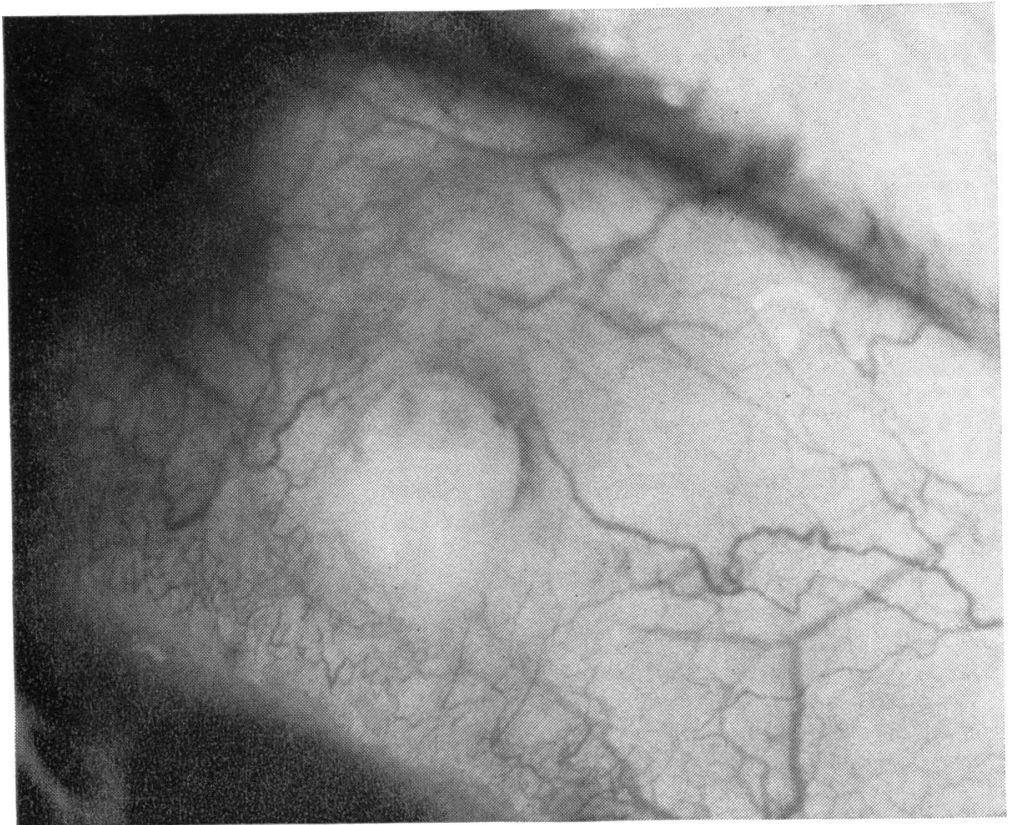

Fig. 7 Case of acute necrotising scleritis

of Bowman's membrane, which sends branches superficially between the epithelial cells (Thomas, 1955).

Any one corneal nerve supplies up to $25 \%$ of the corneal surface. From this description it follows that a lesion behind the perilimbal plexus would have to be extensive to have much effect on sensation if complete interruption of nerve conduction were to occur, and that even a lesion at the limbus or corneal periphery would need to be deep and extensive. It is known from experiments on rabbits (Zander and Weddell, 1951; Rexed and Rexed, 1951), in which different sizes of corneal incision were made, that sensation was lost only adjacent to the wound (Fig. 6). This would be explicable on the basis given above if the corneal nerve supply was similar in the rabbit.

If more than 1 quadrant of the sclera were to undergo inflammation the effect on corneal sensation would be more likely to occur, because adjacent nerves would be unable to compensate. This was found to be the case.

Necrotising scleritis is a rare and devastating form
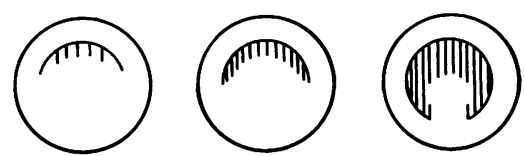

Fig. 6 Loss of corneal sensation resulting from differing sizes of corneal wound of scleritis which has important systemic associations, mainly acute connective tissue disease. In this study 5 cases of necrotising scleritis were examined, and in every one of them there was a profound loss of sensitivity in the corresponding area of the cornea irrespective of the number of quadrants involved. Fig. 7 shows a case of necrotising scleritis which went on to form an area of scleral necrosis that healed after high doses of systemic steroids, leaving an area of scleral ectasia (Fig. 8). Fig. 9 shows the corresponding sensitivity chart during the acute phase. There is an overall decrease in sensitivity in this eye, most marked in the corresponding quadrant. After resolution of this lesion the sensitivity returned to normal.

Necrotising scleritis consists of a granuloma, the centre of which is necrotic, surrounded by an area of oedema and cellular infiltration. Nerves passing through the centre of the affected area would also become necrotic and would not recover. Nerves passing through sclera adjacent to the granuloma would suffer compression and temporary loss of function but would recover when the inflammatory process resolved with treatment. Permanent loss of sensation would result if the area of necrosis was large enough to make adjacent nerves unable to compensate.

The testing of corneal sensitivity in cases of episcleritis and scleritis is a worthwhile investigation. Significant decrease in corneal sensitivity indicates that the inflammatory process is more likely to be a 


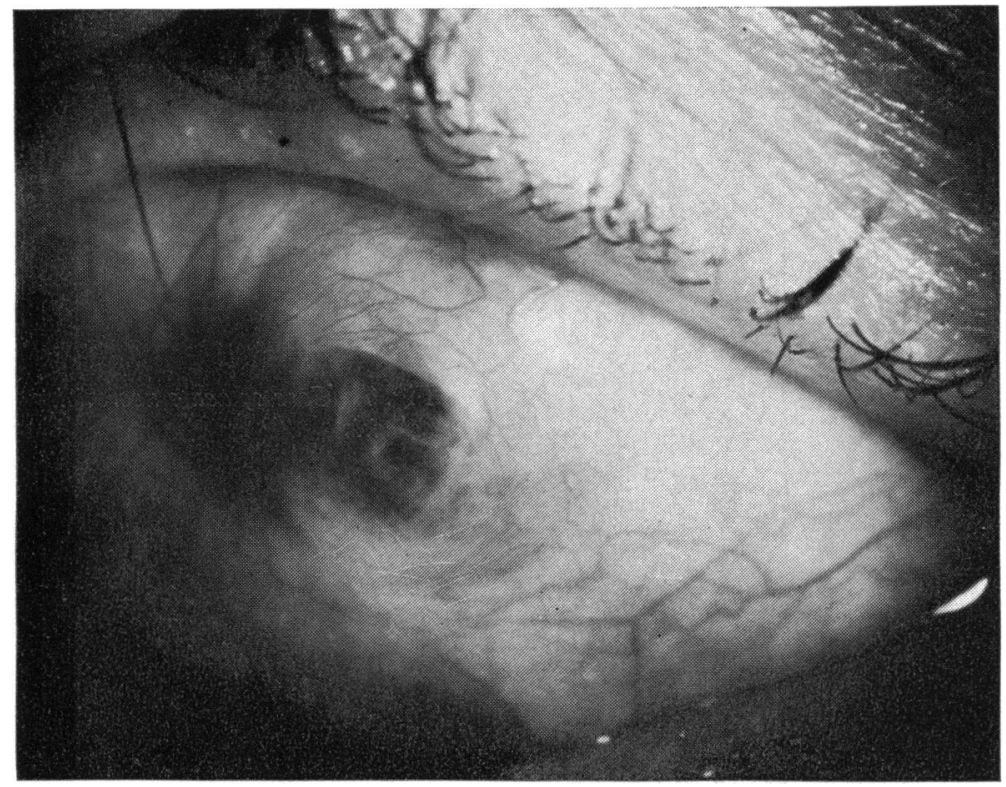

Fig. 8 The same case after treatment in an inactive phase but showing scleral ectasia

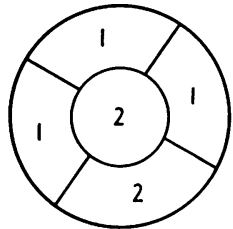

Affected eye

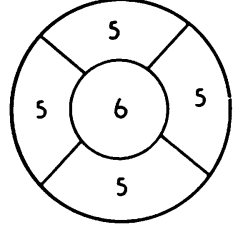

Unoffected eye
Fig. 9 Sensitivity chart of the patient with acute necrotising scleritis

scleritis than an episcleritis. Profound loss of sensation should arouse suspicion of necrotising disease, which would call for immediate treatment with high doses of systemic steroids.

Testing of corneal sensitivity has the advantage that it requires no elaborate equipment, but it does need attention to the points already described in order to get reproducible results. It is advisable to repeat the test at regular intervals to check the validity of the initial findings and as a monitor of the success or otherwise of treatment. Eyes in which the scleritis is resolving show a return to normal corneal sensitivity. This was so even in the presence of so-called scleral thinning. This latter state is usually one of increased scleral transparency (Rooney et al., 1974), in which there is an altered arrangement of the scleral fibres. The only cases showing altered sensation in the quiescent state were those displaying large ectatic areas. These cases were often preceded by a necrotising scleritis, which had presumably damaged corresponding nerves beyond regeneration, and the intraocular pressure was often raised, which itself is known to decrease corneal sensation (Boberg-Ans, 1955).

I thank those surgeons who referred patients to the Scleritis Clinic, Mr P. G. Watson for suggestions with the study, and Miss D. Adamson for help with the typescript.

\section{References}

Boberg-Ans, J. (1955). Experience in clinical examination of corneal sensitivity: Corneal sensitivity and the nasolacrimal reflex after retrobulbar anaesthesia. British Journal of Ophthalmology, 39, 705-728.

Boberg-Ans, J. (1956). On the corneal sensitivity. Acta Ophthalmologia, 34, 149-162.

Marsh, R. J. (1973). Herpes zoster keratitis. Transactions of the Ophthalmological Societies of the United Kingdom, 93, 181-192.

Millodot, M. (1972). Diurnal variation of corneal sensitivity. British Journal of Ophthalmology, 56, 844-847.

Millodot, M., and Lamont, A. (1974). Influence of menstruation on corneal sensitivity. British Journal of Ophthalmology, 58, 752-756.

Rexed, B., and Rexed, U. (1951). Degeneration and regeneration of corneal nerves. British Journal of Ophthalmology, 35, 38-49.

Rooney, P., McGavin, D. M., Sutherland, G. R., and Railton, R. (1974). Thin sclerae: A clinical illusion. Heberden Society: London.

Thomas, C. I. (1955). The Cornea, p. 31. Charles C Thomas: Springfield, Illinois.

Watson, P. G., and Hazleman, B. (1976). The Sclera and Systemic Disease. W. B. Saunders: Eastbourne.

Zander, E., and Weddell, G. (1951). Reaction of corneal nerve fibres to injury. British Journal of Ophthalmology, 35, 61-88. 